\title{
ANALYZING THE TWO-STAGE STRUCTURE OF NARROWBAND IIR FILTERS
}

\author{
Vladimir V. Vityazev, Ruslan S. Goriushkin \\ Department of Telecommunications and Radio Engineering of Ryazan State \\ Radio Engineering University \\ Gagarina Str. 59/1, Ryazan 390005, Russia
}

The paper is received on April 25, 2019

\begin{abstract}
The usage of digital filters with infinite impulse response (IIR) allows reducing computing and hardware costs significantly. However, the instability of such filters prevents their wide usage. This problem is obvious when creating narrowband filters under conditions of limited hardware resources and limited capacity of digital filter coefficients. This work aims to analyze the stability of a twostage form, in particular an interpolated IIR filter and a smoothing filter with an infinite impulse response.
\end{abstract}

Keywords: digital signal processing; narrowband digital filter; IIR-filter; interpolated filter; smoothing filter; multistage filter.

\section{Introduction}

The usage of digital IIR-filters allows reducing computing and hardware costs significantly. The main drawback of such filters is their instability, which prevents their wide adoption. This problem is obvious when creating narrowband filters under conditions of limited hardware resources and limited capacity of digital filter coefficients.

The main way, which is usually used to solve the problem, is transition to a structure of sequential-connected first and second order filters [1]. In this case, the minimum required order of a filter increases, but it also becomes possible to reduce the bit depth of the coefficients [2]. However, the direct form of implementation ismore convenient for digital signal processors (DSP) and programmable logic devices such a field-programmable gate arrays (FPGA).

According to [3], the structure of two series-connected filters shown in [4] 
allows to solve the problem of inaccuracy in the coefficients representation.

This work aims to analyze the stability of a two-stage form, in particular an interpolated IIR filter and a smoothing filter with an infinite impulse response. The analysis was performed with varying narrowband and the bandwidth shape factors of the amplitude-frequency characteristic of a filter. A comparison of two-stage and direct forms is also presented.

\section{Two-stage digital form of an IIR-filter}

An interpolated infinite impulse response (IIIR) filter is a recursive filter where the current output depends on previous outputs. The equation is shown below:

$$
y(n)=\sum_{r=0}^{N_{1}=N / v} b_{r} x[(n-v r) T]-\sum_{k=1}^{M_{1}=M / v} a_{k} y[(n-v r) T]
$$

where $a_{k}$ and $b_{r}$ are the coefficients of a kernel low-pass IIR filter without interpolation that consequently determine the filter characteristics.

To convert a kernel low-pass filter (KF) to an IIIR one, the $z^{-1}$ delay elements must be replaced by the $\mathrm{z}^{-v}$ buffers [5]. The amount of memory required to implement the filter will increase by the $v$ factor.

On the other hand, when an interpolated filter is used, it's possible to filter simultaneously $v$ input signal bands with a bandwidth that is $v$ times smaller than a kernel low-pass filter bandwidth.

As shown in [3], the use of an IIIR filter significantly reduces effects of the limited bit depth on the accuracy of reproducing the frequency characteristics of an interpolated filter with respect to a passband of a kernel low-pass filter. Also, the poles sensitivity to the limited bit depth of an interpolated filter is completely determined by the sensitivity of the poles of a kernel filter.

Let's consider the design of a two-stage low-pass filter. The structure of a designed low-pass filter of the N-th order with the desired $\mathrm{H}(\omega)$ characteristic is represented in the form of a cascade connection of an interpolated IIR filter with the $\mathrm{H}_{\text {IIIR }}(\omega)$ periodic frequency characteristic and a digital smoothing filter with the $\mathrm{H}_{\mathrm{SF}}(\omega)$ frequency characteristic. The components of the $\mathrm{H}_{\mathrm{IIIR}}(\omega)$ repeat the frequency 
selectivity properties of the narrowband low-pass filter by $v$ times within the operating frequency range $0 \leq \omega \leq 2 \pi$. The smoothing filter (SF) selects the main lobe located in the zero frequency area, from the set of lateral components at the output of the IIIR filter $[6,7]$.

The general view of the structure described above is shown in Figure 1. Figure 2 shows the frequency characteristics of the designed low-pass filter as well of the digital IIIR and smoothing filters.

As mentioned above, the poles sensitivity of the digital interpolated filter is approximately $v^{M-1}$ times smaller than the poles sensitivity of the non-cascade filter. A pole shift from the calculated position determines the reproduction accuracy of the desired frequency characteristics.

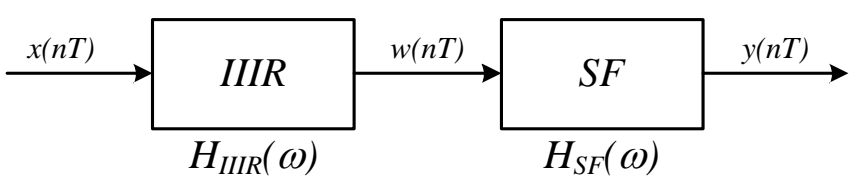

Fig. 1. - Two stage structure of a narrowband IIR filter

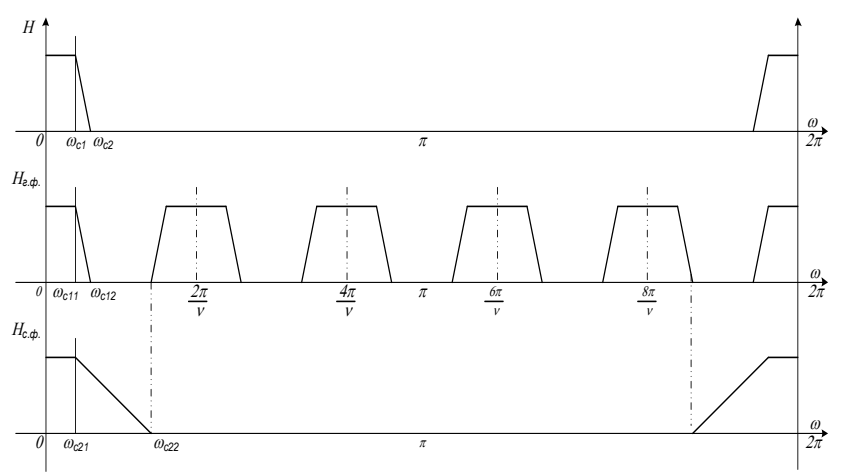

Fig. 2. Amplitude-frequency characteristics of the equivalent LP filter, IIR- and SF-filters

At the same time, the poles sensitivity of the smoothing filter is also significantly smaller than the poles sensitivity of the filter providing the required properties of the $\mathrm{H}(\omega)$ frequency selectivity within the framework of a non-cascade structure. This is due to the fact that the measure of the poles sensitivity significantly depends on the M order of the designed IIR filter and the SF order noticeably decreases when the transition zone of the amplitude-frequency characteristic expands. 


\section{Simulation parameters}

The research was performed by comparing two successively connected IIR filters (interpolated and smoothing) with an equivalent low-pass filter with the similar frequency characteristics.

Filters were designed using fixed values of the passband ripple, stopband attenuation and bandwidth shape factor of the amplitude-frequency characteristic, which can be described as follows:

$$
\alpha=\frac{f_{\text {pass }}}{f_{\text {stop }}}
$$

where $f_{\text {pass }}$ is a passband end and $f_{\text {stop }}$ is a stopband beginning.

The analysis of the structures was performed using variable narrowband coefficients:

$$
\beta=\frac{f_{s}}{f_{\text {pass }}},
$$

where $f_{s}$ is a sampling frequency of input signal.

Each individual filter was designed in Matlab.

\section{Simulation results}

\section{a. Butterworth filters}

Let's consider a Butterworth low-pass filter with the following parameters: input signal sampling frequency $f_{s}=10 \mathrm{kHz}$, stopband attenuation $A_{\text {stop }}=80 \mathrm{~dB}$, passband ripple level $A_{\text {pass }}=0.01$ and bandwidth shape factor $\alpha=0.5$.

We designed Butterworth filters with different values of the narrowband coefficient: $\beta=10 \ldots 1000$. The values of the cutoff frequencies and the orders of the obtained low-pass filters are given in Table 1.

In the Table " $\boldsymbol{x}$ " signs indicate that it's impossible to design a stable filter with the given characteristics in Matlab. The " $\checkmark "$ signs show that a filter can be successfully implemented. In this case, the maximum narrowband coefficient at which an IIR filter can be designed providing the direct form is $\beta=20$. The filter coefficients are represented in a double precision format. However, if the accuracy of representing coefficients is limited to 32 bits $\left(c_{32}\right)$, a stable filter with the given 
characteristics can be calculated only up to $\beta=10$. If the bit depth is limited to 16 bits $\left(\mathrm{c}_{16}\right)$, the calculation is impossible (Table 1).

We designed a two-stage filter with the same parameters, which includes Butterworth interpolated and smoothing filters.

Table 1. Butterworth filter, direct form

\begin{tabular}{|c|c|c|c|c|c|c|}
\hline$\beta$ & 10 & 20 & 40 & 50 & 100 & 200 \\
\hline$f_{\text {pass }}$ & 1000 & 500 & 250 & 200 & 100 & 50 \\
\hline$f_{\text {stop }}$ & 2000 & 1000 & 500 & 400 & 200 & 100 \\
\hline$M$ & 16 & 18 & $\times$ & $\times$ & $\times$ & $\times$ \\
\hline $\mathrm{c}_{16}$ & $\times$ & $\times$ & $\times$ & $\times$ & $\times$ & $\times$ \\
\hline $\mathrm{c}_{32}$ & $\checkmark$ & $\times$ & $\times$ & $\times$ & $\times$ & $\times$ \\
\hline
\end{tabular}

Table 2. Butterworth filter, two-stage form

\begin{tabular}{|c|c|c|c|c|c|c|c|}
\hline$\beta$ & 10 & 20 & 40 & 50 & 100 & 200 & 250 \\
\hline$f_{\text {pass }}$ & 1000 & 500 & 250 & 200 & 100 & 50 & 40 \\
\hline$f_{\text {stop }}$ & 2000 & 1000 & 500 & 400 & 200 & 100 & 80 \\
\hline$v$ & 2 & 4 & 5 & 10 & 10 & 20 & 40 \\
\hline$M_{\mathrm{KF}}$ & 9 & 9 & 14 & 9 & 9 & 11 & 12 \\
\hline $\mathrm{c}_{16}$ & $\checkmark$ & $\checkmark$ & $\times$ & $\times$ & $\times$ & $\times$ & $\times$ \\
\hline $\mathrm{c}_{32}$ & $\checkmark$ & $\checkmark$ & $\checkmark$ & $\checkmark$ & $\checkmark$ & $\checkmark$ & $\checkmark$ \\
\hline
\end{tabular}

Table 2 shows the results of implementing interpolated Butterworth filters. The $v$ periodicity index of the amplitude-frequency characteristic of the interpolated filter varies according to the condition of minimizing the smoothing filter order.

Note that Table 2 shows the kernel filters orders. The smoothing filters orders are within the range $\mathrm{N}=9 . .12$. It can be seen that if the $\beta$ narrowband coefficient is less than 250 and the bit depth is limited to 32 bits, it is possible to design a stable filter with an infinite impulse response in Matlab. If the bit depth is limited to 16 bits, 
the implementation is possible up to $\beta=20$.

\section{b. Elliptic filters}

Elliptic low-pass filters were analyzed the same way.

We designed filters with the same characteristics as used to calculate Butterworth filters: input signal sampling frequency $f_{s}=10 \mathrm{kHz}$, stopband attenuation $A_{\text {stop }}=80 \mathrm{~dB}$, passband ripple level $A_{\text {pass }}=0.01$ and bandwidth shape factor $\alpha=0.5$. The results are summarized in Table 3.

Table 3. Elliptic filter $(\alpha=0.5)$, direct form

\begin{tabular}{|c|c|c|c|c|c|c|}
\hline$\beta$ & 10 & 20 & 40 & 50 & 200 & 250 \\
\hline$f_{\text {pass }}$ & 1000 & 500 & 250 & 200 & 50 & 40 \\
\hline$f_{\text {stop }}$ & 2000 & 1000 & 500 & 400 & 100 & 80 \\
\hline$M$ & 7 & 7 & 7 & 7 & 7 & 7 \\
\hline $\mathrm{c}_{16}$ & $\checkmark$ & $\mathrm{x}$ & $\mathrm{x}$ & $\mathrm{x}$ & $\mathrm{x}$ & $\times$ \\
\hline $\mathrm{c}_{32}$ & $\checkmark$ & $\checkmark$ & $\mathrm{x}$ & $\mathrm{x}$ & $\mathrm{x}$ & $\mathrm{x}$ \\
\hline
\end{tabular}

Table 4. Elliptic filter $(\alpha=0.5)$, two-stage form

\begin{tabular}{|c|c|c|c|c|c|c|c|}
\hline$\beta$ & 10 & 20 & 40 & 50 & 100 & 200 & 250 \\
\hline$f_{\text {pass }}$ & 1000 & 500 & 250 & 200 & 100 & 50 & 40 \\
\hline$f_{\text {stop }}$ & 2000 & 1000 & 500 & 400 & 200 & 100 & 80 \\
\hline$v$ & 2 & 4 & 4 & 4 & 4 & 4 & 4 \\
\hline$M_{\mathrm{KF}}$ & 5 & 5 & 7 & 5 & 5 & 5 & 5 \\
\hline $\mathrm{c}_{16}$ & $\checkmark$ & $\checkmark$ & $\times$ & $\times$ & $\times$ & $\times$ & $\times$ \\
\hline $\mathrm{c}_{32}$ & $\checkmark$ & $\checkmark$ & $\checkmark$ & $\checkmark$ & $\checkmark$ & $\checkmark$ & $\times$ \\
\hline
\end{tabular}

When using an elliptic-type filter, it is possible to implement a digital filter with the narrowband index equal to 250 . If the values are higher, it's impossible to design a stable filter. When limiting the coefficients bit depth, a stable IIR filter with 
the specified parameters can be designed providing $\beta=20$ if the bit depth is 32 bits and $\beta=10$ if the bit depth is 16 bits.

Table 4 shows the results obtained for a two-stage elliptical filter.

A two-stage connection of elliptic filters allows designing a more narrowband structure. In this case, the smoothing filters order shall not exceed 7. An elliptical filter allows designing stable filters even if high narrowband coefficients are required. c. Elliptic filters with an increased bandwidth shape factor

If the $\alpha$ bandwidth shape factor is increased to 0.9 and other parameters stay unchanged, the maximum $\beta$ value reached by a direct form elliptic filter is 20 (Table $5)$.

Table 5. Elliptic filter $(\alpha=0.9)$, direct form

\begin{tabular}{|c|c|c|c|c|c|c|c|}
\hline$\beta$ & 10 & 20 & 40 & 50 & 100 & 200 & 250 \\
\hline$f_{\text {pass }}$ & 1000 & 500 & 250 & 200 & 100 & 50 & 40 \\
\hline$f_{\text {stop }}$ & 1100 & 550 & 275 & 220 & 110 & 55 & 44 \\
\hline$M$ & 13 & 13 & $x$ & $x$ & $x$ & $x$ & $x$ \\
\hline $\mathrm{c}_{16}$ & $x$ & $x$ & $x$ & $x$ & $x$ & $x$ & $x$ \\
\hline$c_{32}$ & $x$ & $x$ & $x$ & $x$ & $x$ & $x$ & $x$ \\
\hline
\end{tabular}

In this case, the limiting of the coefficients bit depth to 32 bits does not allow implementing a stable filter with the given parameters.

Table 6. Elliptic filter $(\alpha=0.9)$, two-stage realization

\begin{tabular}{|c|c|c|c|c|c|c|c|}
\hline$\beta$ & 10 & 20 & 40 & 50 & 100 & 200 & 400 \\
\hline$f_{\text {pass }}$ & 1000 & 500 & 250 & 200 & 100 & 50 & 25 \\
\hline$f_{\text {stop }}$ & 1100 & 550 & 275 & 220 & 110 & 55 & 27 \\
\hline$M_{\mathrm{KF}}$ & 12 & 12 & 12 & 12 & 13 & 13 & 13 \\
\hline$v$ & 2 & 4 & 5 & 10 & 20 & 20 & 40 \\
\hline $\mathrm{c}_{16}$ & $\times$ & $\times$ & $\times$ & $\times$ & $\times$ & $\times$ & $\times$ \\
\hline $\mathrm{c}_{32}$ & $\checkmark$ & $\checkmark$ & $\checkmark$ & $\checkmark$ & $\times$ & $\times$ & $\times$ \\
\hline
\end{tabular}


As in previous cases, the connection of interpolated and smoothing filters makes it possible to design a stable system with $\beta$ equal to 400 (Table 6). When the coefficients bit depth is limited to 32 bits, it is only possible to implement a system with the $\beta$ factor of up to 50 . If the bit depth is limited to 16 bits, it's impossible to design a stable structure.

\section{Conclusion}

The conducted researches show that the use of a two-stage connection of interpolated IIR and smoothing filters allows designing more narrowband filtration systems and reducing their sensitivity to inaccuracy in the coefficients representation. However, this is achieved by increasing the memory resources used to build an IIIR filter. If the narrowband coefficient is low, there is no advantage from using a twostage structure as compared to a direct form low-pass filter. Moreover, the direct form implementation of a stable low-pass filter with a high narrowband coefficient is often impossible. So, we will dedicate the follow-up study to designing structures with more stages.

\section{References}

1. B.A.Shenoi. Introduction to Digital Signal Processing and Filter Design. WileyInterscience, 2005

2. V.V.Vityazev. Mnogoskorostnaja obrabotka signalov [Multirate signal processing], Moscow, Gorjachaja linija - Telekom Publ., 2017. (In Russian)

3. V.V.Vityazev, R.S.Goriushkin. Issledovanie ustojchivosti cifrovyh grebenchatyh fil'trov [Analysing stability of the digital interpolated filters]. 17th International Conference «Digital Signal Processing And Its Applications - DSPA 2015», Moscow, Vol.1, pp 135-138.

4. V.V.Vityazev, S.I.Murav'yev, A.I.Stepashkin. Method of Designing Digital Passband Filters With an Infinite Memory. Telecommunications and Radio Engineering (English translation of Elektrosvyaz and Radiotekhnika), 1984, Vol. 38-9, No. 11, pp. 53-57, 1984 
5. R. Lyons. Improved Narrowband Low-Pass IIR Filters in Fixed-Point Systems. IEEE Signal Processing Magazine, 2009, Vol 5, pp. 128-132

6. T. A. Ramstad, T. Saramaki. Multistage, Multirate FIR Filter Structures for Narrow Transition-Band Filters Proc. 1988, IEEE Int Symp. Circuits and Systems - ISCAS, New Orleans, Louisians, 2017-2021

7. R. Lyons. Interpolated narrowband low-pass FIR filters. IEEE Signal Processing Magazine, 2003, Vol. 20, No. 1, pp. 50-57

\section{For citation:}

Vladimir V. Vityazev, Ruslan S. Goriushkin. Analyzing the Two-Stage Structure of Narrowband IIR Filters. Zhurnal Radioelektroniki - Journal of Radio Electronics. 2019. No.5. Available at http//jre.cplire.ru/jre/may19/7/text.pdf

DOI 10.30898/1684-1719.2019.5.7 